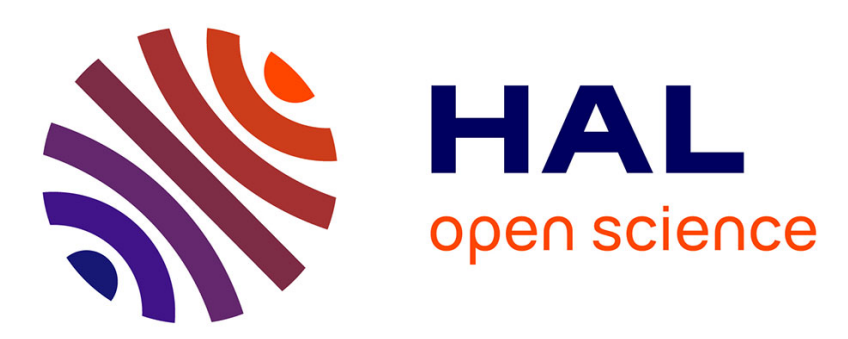

\title{
Controller and observer designs for a class of TS descriptor systems with pole placement constraint
}

Benoît Marx, José Ragot

\section{To cite this version:}

Benoît Marx, José Ragot. Controller and observer designs for a class of TS descriptor systems with pole placement constraint. 45th IEEE Conference on Decision and Control, CDC 2006, Dec 2006, San Diego, CA, United States. pp.6355-6360, 10.1109/CDC.2006.377044 . hal-00121555

\section{HAL Id: hal-00121555 \\ https://hal.science/hal-00121555}

Submitted on 25 Nov 2021

HAL is a multi-disciplinary open access archive for the deposit and dissemination of scientific research documents, whether they are published or not. The documents may come from teaching and research institutions in France or abroad, or from public or private research centers.
L'archive ouverte pluridisciplinaire HAL, est destinée au dépôt et à la diffusion de documents scientifiques de niveau recherche, publiés ou non, émanant des établissements d'enseignement et de recherche français ou étrangers, des laboratoires publics ou privés. 


\title{
Controller and observer designs for a class of TS descriptor systems with pole placement constraint
}

\author{
B. Marx and J. Ragot
}

\begin{abstract}
This paper gives sufficient conditions for poleclustering in LMI regions for a class of TS descriptor systems. First, the class of TS descriptor systems under study is discussed and it is shown to be generic. A strict LMI characterization of pole-clustering is given. Using this new characterization, the design of state-feedback controllers is proposed, in order that the finite dynamics of the closed-loop system lie in a specified LMI region. The design of observer such that the state estimation error dynamics are in a specified LMI region is also studied. Finally, an observer-based controller with pole clustering constraint on the closed-loop system is proposed. A numerical example illustrates the note.
\end{abstract}

\section{INTRODUCTION}

The Takagi-Sugeno (TS) model proposed by [7] is a wellknown method to represent nonlinear systems into several linear fuzzy models. In the last two decades, the control and observation of TS systems has become a challenging problem that received a considerable amount of attention. In [14], stability analysis and controller design is addressed, solutions are derived in the LMI formalism. Relaxed sufficient conditions for fuzzy controllers and fuzzy observers are proposed in [9], and in [8] via a multiple Lyapunov function approach.

The descriptor formalism is also very attractive for system modeling, as pointed in [3], since it describes a wider class of systems than usual systems, including physical systems with non dynamic constraints (e.g. algebraic relations induced in interconnected systems such as power transfer network or water distribution network) or jump behavior. The enhancement of modeling ability is due to the structure of the dynamical equation which encompasses not only dynamic equations, but also algebraic relations. Strict LMI conditions for stabilization $H_{\infty}$ and $H_{2}$ control of descriptor systems are established in [13] and [4] respectively.

The TS representation has been generalized to descriptor systems in [10], [11] and [12]. The stability and the design of state-feedback controllers for T-S descriptors systems (TSDS) are characterized via LMI in [10],[12], the particular problem of nonlinear model following is treated in [12]. Robust output feedback, and $H_{\infty}$ control are considered for TSDS in [5] and [17] respectively. The descriptor formalism is used by [16] for $H_{\infty}$ control of time delay TS systems.

It is well known that the pole location cannot have the same meaning for TS systems than in linear system theory, in particular the stability of the each of the system vertice is

The authors are with the Institut National Polytechnique de Lorraine, CRAN UMR 7039 CNRS-INPL-UHP 2 avenue de la forêt de Haye, 54516 Vandoeuvre-les-Nancy Cedex, FRANCE \{benoit.marx, jose. ragot densem. inpl-nancy. fr not a sufficient condition for the stability of the polytopic system, except if the Lyapunov matrix common for all vertices [1]. A popular solution to deal with this restriction is to seek a Lyapunov function to prove the stability of the system. Nevertheless in the case of systems with different operating modes, remaining in a given mode or a given linear combination of modes for a sufficiently long time, the linear meaning of pole location is recovered. In this case, the stability is not secured during the switching time. In this paper sufficient LMI conditions for finite pole clustering of TSDS are established. The results are expressed in terms of strict LMI conditions, i.e. without equality constraint, and thus are highly tractable and reliable because round-off error while evaluating the equality constraint are avoided [13]. The design of state-feedback controllers is derived from the pole-clustering characterization. The case of uncertain TSDS (constant but unknown activating functions) is envisaged for state feedback. Using a similar method, the design of observer, with pole clustering constraint of the estimation error dynamics is proposed. Finally, estimation and statefeedback control are combined to obtain an observer-based controller for TSDS. The note is written in the continuous time formalism, but the results can be applied to discrete time systems, by choosing different LMI regions.

\section{PROBLEM FORMULATION}

In this section, we introduce the Takagi Sugeno descriptor systems. This class of systems is defined by the following algebraic differential equations, where $x(t) \in \mathbb{R}^{n}$ is the state variable, $u(t) \in \mathbb{R}^{n_{u}}$ is the control input, and $y(t) \in \mathbb{R}^{m}$ is the measured output.

$$
\begin{aligned}
\sum_{i=1}^{N} \mu_{i}(z(t)) E_{i} \dot{x}(t) d t & =\sum_{i=1}^{N} \mu_{i}(z(t))\left(A_{i} x(t)+B_{i} u(t)\right) \\
y(t) & =\sum_{i=1}^{N} \mu_{i}(z(t)) C_{i} x(t)
\end{aligned}
$$

The weighting functions denoted $\mu_{i}(z(t))$ are normalized and verify the following properties

$$
\sum_{i=1}^{N} \mu_{i}(z(t))=1, \quad 0 \leq \mu_{i}(z(t)) \leq 1
$$

Hereafter, it is assumed that the variables $z(t)$ are real time available, and thus so are the weighting functions $\mu_{i}(z(t))$.

The matrices $E_{i}$ are of the form $E_{i}=E_{L} \bar{E}_{i} E_{R}^{T}$, where the matrices $\bar{E}_{i} \in \mathbb{R}^{r \times r}$ are invertible, and the matrices $E_{L}$ and $E_{R} \in \mathbb{R}^{n \times r}$ are of full column rank. This class of matrices 
$E_{i}$ implies that all the subsystems $\left(E_{i}, A_{i}\right)$ have the same differential structure, but allows parameter variation which is usually the case in physical systems. Moreover this approach avoid the state augmentation made in [5], [10], [12], [15] where the state vector $x(t)$ is augmented with its time derivative, which can introduce impulsive mode (obviously, the continuity of $x(t)$ does not implies the continuity of $\dot{x}(t)$.

Most of the previous works concerning TS descriptor systems [5], [10], [12], [15] are based on the transformation of $\left(E_{i}, A_{i}, B_{i}, C_{i}\right)$ into $\left(E_{i}^{*}, A_{i}^{*}, B_{i}^{*}, C_{i}^{*}\right)$. The system (1-2) is then written under the pretended equivalent following form

$$
\begin{aligned}
E^{*} \dot{x}^{*}(t) & =\sum_{i=1}^{N} h_{i}(z(t))\left(A_{i}^{*} x^{*}(t)+B_{i}^{*} u(t)\right) \\
y(t) & =\sum_{i=1}^{N} h_{i}(z(t)) C_{i}^{*} x^{*}(t)
\end{aligned}
$$

where $x^{*}, E^{*}, A_{i}^{*}, B_{i}^{*}, C_{i}^{*}$ are given by

$$
\begin{gathered}
x^{*}=\left[\begin{array}{c}
x \\
\dot{x}
\end{array}\right] \quad E^{*}=\left[\begin{array}{cc}
I_{n} & 0 \\
0 & 0
\end{array}\right] \quad A_{i}^{*}=\left[\begin{array}{cc}
0 & I_{n} \\
A_{i} & -E_{i}
\end{array}\right] \\
B_{i}^{*}=\left[\begin{array}{c}
0 \\
B_{i}
\end{array}\right] \quad C_{i}^{*}=\left[\begin{array}{ll}
C_{i} & 0
\end{array}\right]
\end{gathered}
$$

Unfortunately, this augmentation causes major drawbacks. Unless the matrices $E_{i}$ are full rank (in other words, the pencils-matrices $\left(E_{i}, A_{i}\right)$ are not differential-algebraic, but usual dynamic systems), the pencils-matrices $\left(E^{*}, A_{i}^{*}\right)$ are necessary impulsive. Moreover the systems $\left(E^{*}, A_{i}^{*}, C_{i}^{*}\right)$ are not impulse observable, and the condition for impulse controllability of $\left(E^{*}, A_{i}^{*}, B_{i}^{*}\right)$ is more restrictive than the one concerning the original systems $\left(E_{i}, A_{i}, B_{i}\right)$.

Proposition 1: The following statement are equivalent, for $i=1, \ldots, N$ :

(i) $\operatorname{rank} E_{i}=n$

(ii) the system $\left(E^{*}, A_{i}^{*}\right)$ is impulse free

(iii) the system $\left(E^{*}, A_{i}^{*}, C_{i}^{*}\right)$ is impulse observable

The following statements are equivalent, for $i=1, \ldots, N$ :

(iv) the system $\left(E^{*}, A_{i}^{*}, B_{i}^{*}\right)$ is impulse controllable

(v) $\operatorname{rank}\left[E_{i} \quad B_{i}\right]=n$

Proof: $(i) \Leftrightarrow(i i)$. It is known [3] that $\left(E^{*}, A_{i}^{*}\right) i=$ $1, \ldots, N$ is impulse free if and only if

$$
\begin{aligned}
2 n & =\operatorname{rank}\left[\begin{array}{cc}
E^{*} & A_{i}^{*} \\
0 & E^{*}
\end{array}\right]-\operatorname{rank} E^{*} \\
& =\operatorname{rank}\left[\begin{array}{cccc}
I_{n} & 0 & 0 & I_{n} \\
0 & 0 & A_{i} & -E_{i} \\
0 & 0 & I_{n} & 0 \\
0 & 0 & 0 & 0
\end{array}\right]-n
\end{aligned}
$$

which is obviously equivalent to $\operatorname{rank}_{i}=n$.

(i) $\Leftrightarrow\left(\right.$ iii). The impulse observability of $\left(E^{*}, A_{i}^{*}, C_{i}^{*}\right)$ $i=1, \ldots, N$ is is equivalent to [3]

$$
\begin{aligned}
2 n+\operatorname{rank} E^{*} & =\operatorname{rank}\left[\begin{array}{ccc}
A_{i}^{* T} & E^{* T} & C_{i}^{* T} \\
E^{* T} & 0 & 0
\end{array}\right] \\
& =2 n+\operatorname{rank} E_{i}^{T}
\end{aligned}
$$

which is obviously equivalent to $\operatorname{rank} E_{i}=n$.

$(i v) \Leftrightarrow(v)$. The impulse controllability of $\left(E^{*}, A_{i}^{*}, B_{i}^{*}\right)$ $i=1, \ldots, N$ is equivalent to [3]

$$
\begin{aligned}
2 n+\operatorname{rank} E^{*} & =\operatorname{rank}\left[\begin{array}{ccc}
A_{i}^{*} & E^{*} & B_{i}^{*} \\
E^{*} & 0 & 0
\end{array}\right] \\
& =2 n+\operatorname{rank}\left[\begin{array}{cc}
E_{i} & B_{i}
\end{array}\right]
\end{aligned}
$$

Impulsive terms in the time response of a descriptor system may be highly detrimental for its operation. The impulse controllability (resp. the impulse observability) is the ability to cancel (resp. reconstruct) these undesirable impulsive terms. As a result of the above proposition, if rank $E_{i} \neq n$ the design methods based on the state augmentation introduce impulsive terms which cannot be observed. Moreover the impulse controllability of the original systems $\left(E_{i}, A_{i}, B_{i}\right)$ do not imply the impulse controllability of the systems $\left(E^{*}, A_{i}^{*}, B_{i}^{*}\right)$, since the condition $(v)$ is more restrictive than

$$
\operatorname{rank}\left[\begin{array}{ccc}
A_{i} & E_{i} & B_{i} \\
E_{i} & 0 & 0
\end{array}\right]=n+\operatorname{rank} E_{i}
$$

The previous considerations highlight that most of the results concerning T-S fuzzy descriptor systems are not efficient for algebraic-differential systems but mainly dedicated to descriptor systems with with $\operatorname{rank} E_{i}=n$, which is very restrictive. In such a case all the different systems must be of the same order, despite one of the main interest in T-S fuzzy singular systems is the ability to model systems with different orders behavior.

The aim of this paper is to give some results concerning the analysis, the control and the observation of TSDS with different matrices $E_{i}$ without using the discussed state augmentation.

\section{LMI CHARACTERIZATION POLE CLUSTERING OF TS DESCRIPTOR SYSTEMS}

In this section, the concept of $\mathcal{D}$-admissibility is extended to the TSDS. First, the definition of LMI region and $\mathcal{D}$ admissibility are recalled [2].

Definition 1: A subset $\mathcal{D}$ of the complex plane is called an LMI region if there exists a real symmetric matrix $\alpha \in \mathbb{R}^{p \times p}$ and a matrix $\beta \in \mathbb{R}^{p \times p}$ verifying

$$
\alpha+\beta z+\beta \bar{z}<0, \quad \forall z \in \mathcal{D}
$$

Definition 2: A descriptor system is called $\mathcal{D}$-admissible if it is impulse free, and if its finite poles lie in the LMI region $\mathcal{D}$.

The aim of this section is to give an LMI condition characterizing the $\mathcal{D}$ admissibility of a TSDS. The input free system (12) is governed by a polytopic matrix pencil with vertices defined by the matrices $A_{i}$ and $E_{i}$.

$$
\sum_{i=1}^{N} \mu_{i}(z(t)) E_{i} \dot{x}(t)=\sum_{i=1}^{N} \mu_{i}(z(t)) A_{i} x(t)
$$

To establish the characterization of the $\mathcal{D}$-admissibility of a TSDS, the characterization of the $\mathcal{D}$-admissibility of an LTI descriptor system is needed. 
Lemma 1: [6] The input free LTI descriptor system $E \dot{x}(t)=A x(t)$, where $E$ and $A \in \mathbb{R}^{n \times n}$, is $\mathcal{D}$-admissible if and only if there exists a symmetric positive definite matrix $P \in \mathbb{R}^{n \times n}$ and a matrix $S \in \mathbb{R}^{(n-r) \times(n-r)}$ verifying

$$
\begin{aligned}
\alpha \otimes E P E^{T}+\beta \otimes A P E^{T}+\beta^{T} \otimes E P A^{T} \\
+1_{p p} \otimes\left(A U S V^{T}+V S^{T} U^{T} A^{T}\right)<0
\end{aligned}
$$

where $1_{p p} \in \mathbb{R}^{p \times p}$ denotes the matrix with all entries equal to 1 , and where $U$ and $V \in \mathbb{R}^{n \times(n-r)}$ are full column rank matrices composed of the base of the right null space of $E_{i}$ and $E_{i}^{T}$ respectively.

A sufficient LMI condition for the $\mathcal{D}$-admissibility of (12) is now given.

Theorem 1: The input free TSDS (12) is $\mathcal{D}$-admissible if there exists a symmetric positive definite matrice $P \in \mathbb{R}^{n \times n}$ and a matrice $S \in \mathbb{R}^{(n-r) \times(n-r)}$ verifying the following LMI for $i=1, \ldots, N$ and $j=1, \ldots, N$

$$
\begin{gathered}
\frac{\alpha}{2} \otimes\left(E_{i} P E_{j}^{T}+E_{j} P E_{i}^{T}\right)+\beta \otimes A_{i} P E_{j}^{T}+\beta^{T} \otimes E_{j} P A_{i}^{T} \\
+1_{p p} \otimes\left(A_{i} U S V^{T}+V S^{T} U^{T} A_{i}^{T}\right)<0
\end{gathered}
$$

where $1_{p p} \in \mathbb{R}^{p \times p}$ denotes the matrix with all entries equal to 1 , and where $U$ and $V \in \mathbb{R}^{n \times(n-r)}$ are full column rank matrices composed of the base of the right null space of $E_{i}$ and $E_{i}^{T}$ respectively.

Proof: It is highlighted that common solutions $P$ and $S$ are looked after for all the LMI conditions. Assume there exist $P$ and $S$ verifying (14) for $i=1, \ldots, N$ and $j=$ $1, \ldots, N$. Premultiplying each LMI by $\mu_{i}(z(t)) \mu_{j}(z(t))$, and summing the $N^{2}$ obtained LMI, we have

$$
\begin{aligned}
& \alpha \otimes\left(\sum_{i=1}^{N} \mu_{i}(z(t)) E_{i}\right) P\left(\sum_{j=1}^{N} \mu_{j}(z(t)) E_{j}\right)^{T} \\
& +1_{p p} \otimes \sum_{i=1}^{N} \mu_{i}(z(t))\left(A_{i} U S V^{T}+V S^{T} U^{T} A_{i}^{T}\right) \\
& +\beta \otimes\left(\sum_{i=1}^{N} \mu_{i}(z(t)) A_{i}\right) P\left(\sum_{j=1}^{N} \mu_{j}(z(t)) E_{j}\right)^{T} \\
& +\beta^{T} \otimes\left(\sum_{j=1}^{N} \mu_{j}(z(t)) E_{j}\right) P\left(\sum_{i=1}^{N} \mu_{i}(z(t)) A_{i}\right)^{T}<0
\end{aligned}
$$

which implies the pole clustering of the finite dynamics of (12) for all $t$.

\section{IV. $\mathcal{D}$-STABILIZING STATE FEEDBACK}

In this section, the goal is to determine the gains $K_{k} \in$ $\mathbb{R}^{n_{u} \times n}$ of the following control law

$$
u(t)=\sum_{k=1}^{N} \mu_{k}(z(t)) K_{k} x(t)
$$

in order that the closed-loop system (17) is $\mathcal{D}$-admissible.

$$
\begin{aligned}
\sum_{i=1}^{N} \mu_{i}(z(t)) E_{i} \dot{x}(t) d t= & \sum_{i=1}^{N} \sum_{k=1}^{N} \mu_{i}(z(t)) \mu_{k}(z(t))\left(\left(A_{i}\right.\right. \\
& \left.\left.+B_{i} K_{k}\right) x(t)\right)
\end{aligned}
$$

The controller is designed by solving a set of strict LMI as established in the following theorem.

Theorem 2: For a given LMI region $\mathcal{D}$, defined by $\alpha=$ $\alpha^{T}$ and $\beta$, there exist a control law (16) such that the closedloop system (17) is $\mathcal{D}$-admissible if there exist a symmetric positive definite matrice $P \in \mathbb{R}^{n \times n}$, a non singular matrix $S \in \mathbb{R}^{(n-r) \times(n-r)}$, matrices $L_{k} \in \mathbb{R}^{n_{u} \times(n-r)}$ and $H_{k} \in$ $\mathbb{R}^{n_{u} \times(n-r)}$, verifying the following LMI for $i=1, \ldots, N$, $j=1, \ldots, N$ and $k=1, \ldots, N$.

$$
\begin{gathered}
\frac{\alpha}{2} \otimes\left(E_{i} P E_{j}^{T}+E_{j} P E_{i}^{T}\right)+\beta \otimes\left(A_{i} P E_{j}^{T}+B_{i} L_{k} E_{j}^{T}\right) \\
+\beta^{T} \otimes\left(E_{j} P A_{i}^{T}+E_{j} L_{k}^{T} B_{i}^{T}\right) \\
+1_{p p} \otimes\left(A_{i} U S V^{T}+V S^{T} U^{T} A_{i}^{T}+B_{i} H_{k} V^{T}+V^{T} H_{k}^{T} B_{i}^{T}\right)<0
\end{gathered}
$$

where $1_{p p} \in \mathbb{R}^{p \times p}$ denotes the matrix with all entries equal to 1 , and where $U$ and $V \in \mathbb{R}^{n \times(n-r)}$ are full column rank matrices composed of the base of the right null space of $E_{i}$ and $E_{i}^{T}$ respectively. The gains $K_{k}$ are given by

$$
\begin{aligned}
K_{k}= & \left(L_{k} E_{i}^{T}+H_{k} V^{T}\right)\left(P E_{i}+U S V^{T}\right)^{-1} \\
= & H_{k} S^{-1}\left(U^{T} U\right)^{-1} U^{T}\left(I_{n}-P E_{R}\left(E_{R}^{T} P E_{R}\right)^{-1} E_{R}^{T}\right) \\
& +L_{k} E_{R}\left(E_{R}^{T} P E_{R}\right)^{-1} E_{R}^{T}
\end{aligned}
$$

Proof: Assume there exist matrices $P, S, L_{k}$ and $H_{k}$, verifying the $N^{3}$ LMI (18). Firstly, it is shown that $P E_{i}^{T}+U S V^{T}$ is invertible. Matrices $\bar{E}_{i}$ are invertible, and matrices $E_{R}$ and $E_{L}$ are of full column rank. As a consequence $E_{i} U=0$ and $E_{i}^{T} V=0$ imply $E_{R}^{T} U=0$ and $E_{L}^{T} V=0$ respectively. Thus, we obtain

$$
\left[\begin{array}{c}
\left(E_{L}^{T} E_{L}\right)^{-1} E_{L}^{T} \\
\left(V^{T} V\right)^{-1} V^{T}
\end{array}\right]\left[\begin{array}{ll}
E_{L} & V
\end{array}\right]=I_{n}
$$

The two matrices of the left side of (21) are in $\mathbb{R}^{n \times n}$, and are of full rank. Thus, each is the inverse of the other, and we have

$$
E_{L}\left(E_{L}^{T} E_{L}\right)^{-1} E_{L}^{T}+V\left(V^{T} V\right)^{-1} V^{T}=I_{n}
$$

which implies

$$
\begin{gathered}
\left(E_{L}\left(E_{L}^{T} E_{L}\right)^{-1}\left(\bar{E}_{i}^{T}\right)^{-1}\left(E_{R}^{T} P E_{R}\right)^{-1} E_{R}^{T}\right. \\
+V\left(V^{T} V\right)^{-1} S^{-1}\left(U^{T} U\right)^{-1} U^{T}\left(I_{n}\right. \\
\left.\left.-P E_{R}\left(E_{R}^{T} P E_{R}\right)^{-1} E_{R}^{T}\right)\right)\left(P E_{i}^{T}+U S V^{T}\right)=I_{n}
\end{gathered}
$$

in other words

$$
\begin{aligned}
& \left(P E_{i}^{T}+U S V^{T}\right)^{-1}=E_{L}\left(E_{L}^{T} E_{L}\right)^{-1}\left(\bar{E}_{i}^{T}\right)^{-1}\left(E_{R}^{T} P E_{R}\right)^{-1} E_{R}^{T} \\
& +V\left(V^{T} V\right)^{-1} S^{-1}\left(U^{T} U\right)^{-1} U^{T}\left(I_{n}-P E_{R}\left(E_{R}^{T} P E_{R}\right)^{-1} E_{R}^{T}\right)
\end{aligned}
$$

The gains $K_{k}$ (20), are obtained by substituting (24) in (19). One can verify that (20) implies $K_{k} P E_{i}^{T}=L_{k} E_{i}^{T}$ and $K_{k} U S=H_{k}$. Substituting $K_{k} P E_{i}^{T}$ and $K_{k} U S$ to $L_{k} E_{i}^{T}$ 
and $H_{k}$ respectively, in (18), multiplying by $\mu_{k}(z(t))$, and summing for $k=1, \ldots, N$ the $N$ obtained LMI, the LMI condition (14) is obtained for $\left(E_{j}, A_{i}+B_{i} \sum_{k=1}^{N} \mu_{k} K_{k}\right)$ which achieves the proof.

In the framework of uncertain systems, i.e. when the activating functions are constant but unknown, the state feedback controller can be designed with a unique feedback gain $K$, then the control law is $u(t)=K x(t)$. In this case, common variables $H$ and $L$ are looked after when solving the LMI (20) which becomes, for $i=1, \ldots, N$ and $j=1, \ldots, N$,

$$
\begin{gathered}
1_{p p} \otimes\left(A_{i} U S V^{T}+V S^{T} U^{T} A_{i}^{T}+B_{i} H V^{T}+V^{T} H^{T} B_{i}^{T}\right) \\
+\beta \otimes\left(A_{i} P E_{j}^{T}+B_{i} L E_{j}^{T}\right)+\beta^{T} \otimes\left(E_{j} P A_{i}^{T}+E_{j} L^{T} B_{i}^{T}\right) \\
\frac{\alpha}{2} \otimes\left(E_{i} P E_{j}^{T}+E_{j} P E_{i}^{T}\right)<0
\end{gathered}
$$

And the gain $K$ is given by

$$
\begin{aligned}
K= & H S^{-1}\left(U^{T} U\right)^{-1} U^{T}\left(I_{n}-P E_{R}\left(E_{R}^{T} P E_{R}\right)^{-1} E_{R}^{T}\right) \\
& +L E_{R}\left(E_{R}^{T} P E_{R}\right)^{-1} E_{R}^{T}
\end{aligned}
$$

\section{OBSERVERS DESIGN}

In this section, the design of observers for TSDS is addressed. The proposed observers are in TSDS form, and are defined by

$$
\begin{aligned}
\sum_{i=1}^{N} \mu_{i}(z(t)) E_{i} \dot{\hat{x}}(t) d t & =\sum_{i=1}^{N} \mu_{i}(z(t))\left(A_{i} \hat{x}(t)+B_{i} u(t)\right. \\
& \left.+G_{i}\left(\sum_{j=1}^{N} \mu_{j}(z(t)) C_{j} \hat{x}(t)-y(t)\right)\right) \\
\hat{y}(t) & =\sum_{i=1}^{N} \mu_{i}(z(t)) C_{i} \hat{x}(t)
\end{aligned}
$$

The objective is to determine the gains $G_{i}$ such that the state estimation error is impulse free and that its finite poles lie in the left half complex plane.

Theorem 3: The system (27-28) is an observer for (1-2) if there exist a symmetric positive definite $P \in \mathbb{R}^{n \times n}$, a non singular matrix $S \in \mathbb{R}^{(n-r) \times(n-r)}$, matrices $L_{j} \in \mathbb{R}^{n \times m}$ and $H_{j} \in \mathbb{R}^{(n-r) \times m}$ verifying the following LMI for $i=$ $1, \ldots, N, j=1, \ldots, N$ and $k=1, \ldots, N$.

$$
\begin{aligned}
& A_{j}^{T}\left(P E_{i}+V S U^{T}\right)+\left(E_{i}^{T} P+U S^{T} V^{T}\right) A_{j} \\
+ & C_{k}^{T}\left(L_{j}^{T} E_{i}+H_{j}^{T} U^{T}\right)+\left(E_{i}^{T} L_{j}+U H_{j}\right) C_{k}<0
\end{aligned}
$$

Where $U$ and $V \in \mathbb{R}^{n \times(n-r)}$ are full column rank matrices composed of the base of the right null space of $E_{i}$ and $E_{i}^{T}$ respectively.

The observer gains $G_{j}$ are given by

$$
\begin{aligned}
G_{j}= & \left(E_{i}^{T} P+U S^{T} V^{T}\right)^{-1}\left(E_{i}^{T} L_{j}+U H_{j}\right) \\
= & \left(I_{n}-E_{L}\left(E_{L}^{T} P E_{L}\right)^{-1} E_{L}^{T} P\right) V\left(V^{T} V\right)^{-1}\left(S^{T}\right)^{-1} H_{j} \\
& +E_{L}\left(E_{L}^{T} P E_{L}\right)^{-1} E_{L}^{T} L_{j}
\end{aligned}
$$

Proof: The state estimation error, defined by $e(t)=$ $x(t)-\hat{x}(t)$, is governed by the following algebraic differential relation

$$
\sum_{i=1}^{N} \mu_{i}(z(t)) E_{i} \dot{e}(t) d t=\sum_{i=1}^{N} \sum_{j=1}^{N} \mu_{i j}(z(t))\left(A_{i}+G_{i} C_{j}\right) e(t)
$$

Where $\mu_{i j}(z(t))=\mu_{i}(z(t)) \mu_{j}(z(t))$. The matrices $E_{i}$ and $U$ satisfy $E_{i} U=E_{L} \bar{E}_{i} E_{R}^{T} U=0$, and $E_{L} \bar{E}_{i}$ is of full column rank, which implies : $E_{R}^{T} U=0$. Similarly, $E_{i}^{T} V=0$ implies $E_{L}^{T} V=0$. Simple calculus leads to

$$
\left[\begin{array}{c}
\left(E_{R}^{T} E_{R}\right)^{-1} E_{R}^{T} \\
\left(U^{T} U\right)^{-1} U^{T}
\end{array}\right]\left[\begin{array}{ll}
E_{R} & U
\end{array}\right]=I_{n}
$$

In other words, we have

$$
\begin{aligned}
{\left[E_{R} U\right]\left[\begin{array}{c}
\left(E_{R}^{T} E_{R}\right)^{-1} E_{R}^{T} \\
\left(U^{T} U\right)^{-1} U^{T}
\end{array}\right] } & =E_{R}\left(E_{R}^{T} E_{R}\right)^{-1} E_{R}^{T}+U\left(U^{T} U\right)^{-1} U \\
& =I_{n}
\end{aligned}
$$

From which one can derive

$I=\left(E_{i}^{T} P+U S^{T} V^{T}\right)\left(E_{L}\left(E_{L}^{T} P E_{L}\right)^{-1}\left(\bar{E}_{i}^{T}\right)^{-1}\left(E_{R}^{T} E_{R}\right)^{-1} E_{R}^{T}\right.$ $\left.+\left(I-E_{L}\left(E_{L}^{T} P E_{L}\right)^{-1} E_{L}^{T} P\right) V\left(V^{T} V\right)^{-1}\left(S^{T}\right)^{-1}\left(U^{T} U\right)^{-1} U^{T}\right)$

which can be written as

$$
\begin{aligned}
& \left(E_{i}^{T} P+U S^{T} V^{T}\right)^{-1}=E_{L}\left(E_{L}^{T} P E_{L}\right)^{-1}\left(\bar{E}_{i}^{T}\right)^{-1}\left(E_{R}^{T} E_{R}\right)^{-1} E_{R}^{T} \\
& +\left(I-E_{L}\left(E_{L}^{T} P E_{L}\right)^{-1} E_{L}^{T} P\right) V\left(V^{T} V\right)^{-1}\left(S^{T}\right)^{-1}\left(U^{T} U\right)^{-1} U^{T}
\end{aligned}
$$

Assume that there exist matrices $P, S, L_{j}$ and $H_{j}$ verifying (29), and set

$$
\left(E_{i}^{T} L_{j}+U H_{j}\right)=\left(E_{i}^{T} P+U S^{T} V^{T}\right) G_{j}
$$

Since the matrix $\left(E_{i}^{T} P+U S^{T} V^{T}\right)$ is invertible, determining the matrices $L_{j}$ and $H_{j}$ defines $G_{j}$, and we have

$$
\begin{gathered}
\left(A_{j}^{T}+C_{k}^{T} G_{j}^{T}\right)\left(P E_{i}+V S U^{T}\right) \\
+\left(E_{i}^{T} P+U S^{T} V^{T}\right)\left(A_{j}+G_{j} C_{k}\right)<0
\end{gathered}
$$

Premultiplying (38) by $\mu_{k}(z(t))$ and summing for $k=$ $1, \ldots, N$, the following LMI are obtained

$$
\tilde{A}_{j}^{T}\left(P E_{i}+V S U^{T}\right)+\left(E_{i}^{T} P+U S^{T} V^{T}\right) \tilde{A}_{j}<0
$$

where $\tilde{A}_{i}=\sum_{k=1}^{N} \mu_{k}(z(t))\left(A_{i}+G_{i} C_{k}\right)$. The LMI (39) corresponds to (14) with $\alpha=0$ and $\beta=1$, in other words, $\left(E_{i}, \tilde{A}_{j}\right)$ is $\mathcal{D}$ admissible, where $\mathcal{D}$ is the left half complex plane.

The equivalence between (30) and (31), established with (36), proves that the gains $G_{j}$ do not depend on the indice $i$.

The result of the previous section can be applied to place the finite poles of the equation governing the state estimation error in a specified LMI region, by appropriately choosing the gains $G_{k}$.

Theorem 4: for a given LMI region $\mathcal{D}$, defined by the matrices $\alpha=\alpha^{T}$ and $\beta \in \mathbb{C}^{p \times p}$, the system (27-28) is an observer for (1-2), with the state estimation error dynamic 
located in $\mathcal{D}$ if there exist a symmetric positive definite matrix $P \in \mathbb{R}^{n \times n}$, a non singular matrix $S \in \mathbb{R}^{(n-r) \times(n-r)}$, matrices $L_{j} \in \mathbb{R}^{n \times m}$ and $H_{j} \in \mathbb{R}^{(n-r) \times m}$ verifying the following LMI for $i=1, \ldots, N, j=1, \ldots, N$ and $k=$ $1, \ldots, N$.

$$
\begin{array}{r}
1_{p p} \otimes\left(A_{j}^{T} V S U^{T}+C_{k}^{T} H_{j}^{T} U^{T}+U S^{T} V^{T} A_{j}+U H_{j} C_{k}\right) \\
+\beta \otimes\left(A_{j}^{T} P+C_{k}^{T} L_{j}^{T}\right) E_{i}+\beta^{T} \otimes E_{i}^{T}\left(P A_{j}+L_{j} C_{k}\right) \\
+\frac{\alpha}{2} \otimes\left(E_{i}^{T} P E_{j}+E_{j} P E_{i}^{T}\right)<0
\end{array}
$$

Where $U$ and $V \in \mathbb{R}^{n \times(n-r)}$ are full column rank matrices composed of the base of the right null space of $E_{i}$ and $E_{i}^{T}$ respectively.

The observer gains $G_{j}$ are given by

$$
\begin{aligned}
G_{j}= & \left(E_{i}^{T} P+U S^{T} V^{T}\right)^{-1}\left(E_{i}^{T} L_{j}+U H_{j}\right) \\
= & E_{L}\left(E_{L}^{T} P E_{L}\right)^{-1} E_{L}^{T} L_{j}+\left(I_{n}-E_{L}\left(E_{L}^{T} P E_{L}\right)^{-1} E_{L}^{T} P\right) \\
& V\left(V^{T} V\right)^{-1}\left(S^{T}\right)^{-1} H_{j}
\end{aligned}
$$

Proof: By duality, the proof is similar to the proof of theorem 2, and thus omitted.

A minimal decay rate of the observer can be imposed by choosing the appropriate LMI region. The left half plane defined by $\{z \in \mathbb{C} \mid \Re(z)<-\lambda\}$ is the LMI region defined by $\alpha=2 \lambda$ and $\beta=1$.

Corollary 1: The system (27-28) is an observer for (1-2) with a minimal decay rate of $\lambda$ if there exist a symmetric positive definite matrix $P \in \mathbb{R}^{n \times n}$, a non singular matrix $S \in \mathbb{R}^{(n-r) \times(n-r)}$, matrices $L_{j} \in \mathbb{R}^{n \times m}$ and $H_{j} \in$ $\mathbb{R}^{(n-r) \times m}$ verifying the following LMI for $i=1, \ldots, N$, $j=1, \ldots, N, k=1, \ldots, N$ and $l=1, \ldots, N$.

$$
\begin{aligned}
& \left(A_{j}^{T}+\lambda E_{l}^{T}\right)\left(P E_{i}+V S U^{T}\right)+\left(E_{i}^{T} P+U S^{T} V^{T}\right)\left(A_{j}+\lambda E_{l}\right) \\
& \quad+C_{k}^{T}\left(L_{j}^{T} E_{i}+H_{j}^{T} U^{T}\right)+\left(E_{i}^{T} L_{j}+U H_{j}\right) C_{k}<0
\end{aligned}
$$

Where $U$ and $V \in \mathbb{R}^{n \times(n-r)}$ are full column rank matrices composed of the base of the right null space of $E_{i}$ and $E_{i}^{T}$ respectively.

The observer gains $G_{j}$ are given by (31).

\section{OBSERVER-BASED CONTROL}

In this section, a control law is derived from the state estimation given by the observer (27-28). The control input is defined by

$$
u(t)=\sum_{j=1}^{N} \mu_{j}(z(t)) F_{j} \hat{x}(t)
$$

Substituting (44) in (1), and defining and augmented state vector by $\underline{x}^{T}(t)=\left[x^{T}(t) \hat{x}^{T}(t)\right]$, the closed-loop system can be rewritten as an augmented system given by

$$
\begin{aligned}
\sum_{i=1}^{N} \mu_{i}(z(t)) \underline{E}_{a i} \underline{\dot{x}}(t) & =\sum_{i=1}^{N} \sum_{j=1}^{N} \mu_{i j}(z(t)) \mu_{j}(z(t)) \underline{A}_{a i} \underline{x}(t) \\
y(t) & =\sum_{i=1}^{N} \mu_{i}(z(t))\left[\begin{array}{ll}
C_{i} & 0
\end{array}\right] \underline{x}(t)
\end{aligned}
$$

with

$$
\underline{E}_{a i}=\left[\begin{array}{cc}
E_{i} & 0 \\
0 & E_{i}
\end{array}\right] \quad \underline{A}_{a i}=\left[\begin{array}{cc}
A_{i} & B_{i} F_{j} \\
-G_{i} C_{j} & A_{i}+G_{i} C_{j}+B_{i} F_{j}
\end{array}\right]
$$

Pre-multiplying (45) by $T_{1}$, and defining the new augmented state $x_{a}(t)$ defined

$$
T 1=\left[\begin{array}{cc}
I_{n} & 0 \\
-I_{n} & I_{n}
\end{array}\right] \quad x_{a}(t)=\left[\begin{array}{c}
x(t) \\
\hat{x}(t)-x(t)
\end{array}\right]
$$

the well-known separation principle is recovered, and the closed-loop system becomes

$$
\sum_{i=1}^{N} \mu_{i}(z(t)) \underline{E}_{a i} \dot{x}_{a}(t)=\sum_{i=1}^{N} \sum_{j=1}^{N} \mu_{i}(z(t)) \mu_{j}(z(t)) A_{a i} x_{a}(t)
$$

$$
y(t)=\sum_{i=1}^{N} \mu_{i}(z(t))\left[\begin{array}{ll}
C_{i} & 0
\end{array}\right] x_{a}(t)
$$

with

$$
A_{a i}=\left[\begin{array}{cc}
A_{i} & B_{i} F_{j} \\
-G_{i} C_{j} & A_{i}+G_{i} C_{j}+B_{i} F_{j}
\end{array}\right]
$$

The finite spectrum of the closed-loop system is the reunion of the finite spectrum of $\left(E_{i}, A_{i}+B_{i} F_{j}\right)$ and $\left(E_{i}, A_{i}+\right.$ $G_{i} C_{j}$ ). The determination of the gains of the observer $G_{i}$ and of the control law $F_{j}$ have been discussed in section $\mathrm{V}$ and IV respectively. Solving the LMI (40) and (18) the observer-based controller is designed.

\section{NUMERICAL EXAMPLE}

Due to the space limitation, only the observer design is illustrated. Consider the TSDS (1-2) defined by

$$
\begin{aligned}
E_{1}=\left(\begin{array}{lll}
1 & 1 & 0 \\
0 & 1 & 0 \\
0 & 0 & 0
\end{array}\right) & E_{2}=\left(\begin{array}{lll}
1 & 0 & 0 \\
0 & 1 & 0 \\
0 & 0 & 0
\end{array}\right) \\
A_{1}=\left(\begin{array}{ccc}
-1 & -2 & 0 \\
2 & -3 & 4 \\
0 & 0 & 1
\end{array}\right) & A_{2}=\left(\begin{array}{ccc}
-1 & -2 & 0 \\
2 & -2 & 2 \\
0 & 3 & 1
\end{array}\right) \\
B_{1}=\left(\begin{array}{ll}
2 & 1 \\
1 & 0 \\
0 & 0
\end{array}\right) & B_{2}=\left(\begin{array}{ll}
0 & 1 \\
1 & 0 \\
0 & 0
\end{array}\right) \\
C_{1}=\left(\begin{array}{ccc}
1 & 1 & 0
\end{array}\right) & C_{2}=\left(\begin{array}{lll}
1 & 1 & 0
\end{array}\right) \\
1+\tanh \left(u_{1}(t) / 15\right) & h_{2}(z(t))=1-h_{1}(t)
\end{aligned}
$$

The observer is designed to be $\mathcal{D}$ admissible with $\alpha=10$ and $\beta=1$, in other words, with a minimum decay rate of $\lambda=5$.

$$
\begin{gathered}
P=\left(\begin{array}{ccc}
4.961 & 11.91 & -30.08 \\
11.91 & 37.79 & -124.8 \\
-30.08 & -124.8 & 666.8
\end{array}\right) \quad S=-49.34 \\
G_{1}=\left(\begin{array}{c}
-32.33 \\
7.758 \\
0.3228
\end{array}\right) \quad G_{2}=\left(\begin{array}{c}
-128.8 \\
38.49 \\
-1.689
\end{array}\right)
\end{gathered}
$$

The initial state of the observer $\hat{x}(0)=\left[\begin{array}{lll}-2 & -24\end{array}\right]$, while the initial state of the system is $x(0)=\left[\begin{array}{lll}2 & 2 & -2\end{array}\right]$. Simulation 
results are displayed on the figures 1, 2, 3. Each figure displays the comparison of the state variable and its estimate.

\section{CONCLUSION}

In this note, pole clustering in LMI regions for a class of Takagi-Sugeno descriptor systems is characterized by strict LMI. State feedback controller, full order observer and observer-based controller designs are derived. The determinations of the parameters of the controllers and observer are given in the terms of strict LMI. The results established in this note are valid only for systems with slow variation of the weighting functions.

\section{REFERENCES}

[1] S. Boyd, L. El Ghaoui, E. Feron and V. Balakrishnan, Linear Matrix Inequalities in System and Control Theory, SIAM studies in applies mathematics, 1994.

[2] M. Chilali and P. Gahinet, " $H_{\infty}$-design with pole placement constraints: an LMI approach", IEEE Trans. Autom. Contr., vol. 41, pp. 358-367, 1996.

[3] L. Dai, Singular Control Systems, Springer, Germany, 1989.

[4] M. Ikeda, T.K. Lee and E. Uezato, "A Strict LMI Condition for $\mathrm{H}_{2}$ Control of Descriptor Systems", in Proc. of the $39^{\text {th }}$ IEEE Conference on Decision and Control, pp. 601-604, 2000.

[5] K.Y. Lian, P. Liu, J.J. Liou and T.C. Wu, "Robust Output Feedback Control for Fuzzy Descriptor Systems", in Proc of the IEEE Int. Fuzzy Syst. Conf., vol. 2, pp. 904-907, 2001.

[6] B. Marx, D. Koenig and D. Georges, "Robust Pole-Clustering for Descriptor Systems, a Strict LMI Characterization", in Proc. of the European Control Conference, article 189, 2003.

[7] T. Takagi and M. Sugeno, "Fuzzy Identification of Systems and its Application to Modeling and Control", IEEE Trans. Syst., Man, Cybern., vol. 15, pp. 116-132, 1985.

[8] K. Tanaka, T. Hori and H.O. Wang, "A multiple Lyapunov function approach to stabilization of fuzzy control systems", IEEE Trans. Fuzzy Syst., vol. 11, pp. 582-589, 2003.

[9] K. Tanaka, T. Ikeda and H.O. Wang, "Fuzzy Regulators and Fuzzy Observers: Relaxed Stability Conditions and LMI-Based Designs", IEEE Trans. Fuzzy Syst., vol. 6, pp. 250-265, 1998.

[10] T. Taniguchi, K. Tanaka, K. Yamafuji and H.O. Wang, "Fuzzy Descriptor Systems : Stability Analysis and Design via LMIs ", in Proc. of the Amer. Contr. Conf., vol. 3, pp. 1827-1831, 1999.

[11] T. Taniguchi, K. Tanaka and H.O. Wang, "Fuzzy Descriptor Systems and Fuzzy Controller Designs", in Proc. of the $8^{\text {th }}$ Int. Fuzzy Syst. Assoc. World Congress, Vol. 2, pp. 655-659, 1999.

[12] T. Taniguchi, K. Tanaka and H.O. Wang, "Fuzzy Descriptor Systems and Nonlinear Model Following Control", IEEE Trans. Fuzzy Syst., vol. 8, pp. 442-452, 2000.

[13] E. Uezato and M. Ikeda, Strict LMI Conditions for Stability, Robust Stabilization, and $H_{\infty}$-Control of Descriptor Systems, in Proc. of the $38^{\text {th }}$ IEEE Conference on Decision and Control., pp. 4092-4097, 1999.

[14] H.O. Wang, K. Tanaka, M.F. Griffin, "An Approach to Fuzzy Control of Nonlinear Systems : Stability and Design Issues", IEEE Trans. Fuzzy Syst., vol. 4, pp. 14-23, 1996.

[15] Y. Wang, Q.L. Zhang and W.Q. Liu, "Stability Analysis and Design for T-S Fuzzy Descriptor Systems", in Proc. of the IEEE Conf. Decision Contr., vol. 4, pp. 3962-3967, 2001

[16] J. Yoneyama, " $H_{\infty}$ Control for Fuzzy Time-Delay Systems via Descriptor Approach", in Proc. of the IEEE International Symp. on Intelligent Contr., pp. 407-412, 2004.

[17] J. Yoneyama and I. Ichikawa, " $H_{\infty}$-Control for Takagi-Sugeno Fuzzy Descriptor Systems", in Proc of the IEEE Int. Conf. Syst. Man Cybern., vol. 3, pp. 28-33, 1999.

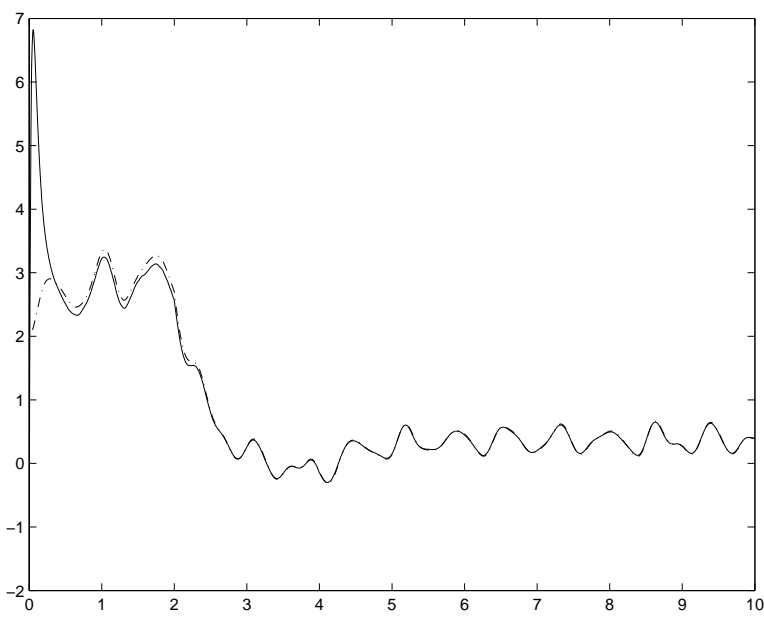

Fig. 1. Comparison of $x_{1}(t)$ (dotted line) and $\hat{x}_{1}(t)$ (solid line)

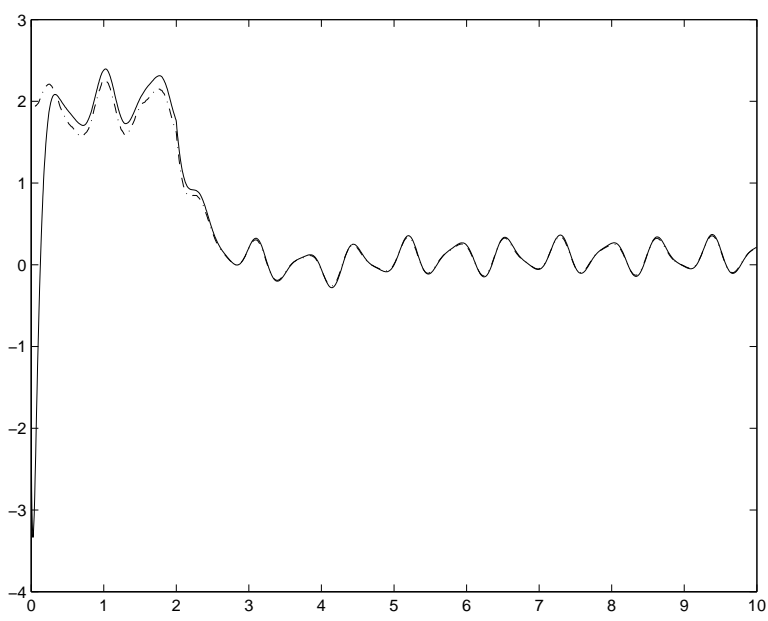

Fig. 2. Comparison of $x_{2}(t)$ (dotted line) and $\hat{x}_{2}(t)$ (solid line)

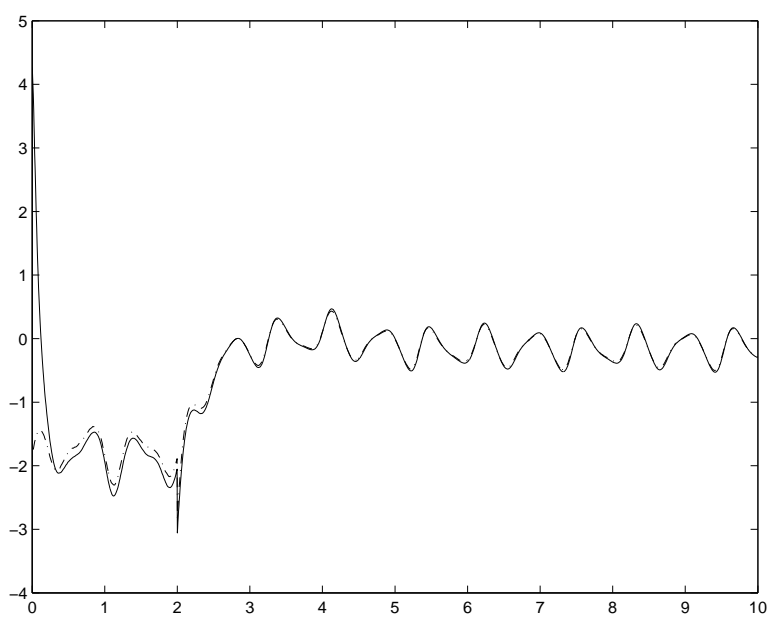

Fig. 3. Comparison of $x_{3}(t)$ (dotted line) and $\hat{x}_{3}(t)$ (solid line) 